\title{
When and to whom should palliative care be offered in heart failure?
}

\author{
Goes GHB ${ }^{1 *}$ and Sobral Filho DC ${ }^{2}$ \\ ${ }^{1}$ Medical Student, University of Pernambuco (UPE), Faculty of Medical Sciences, Cardiac Emergency Hospital of Pernambuco (PROCAPE - UPE), Recife, Brazil \\ ${ }^{2}$ Associate Professor of Cardiology at the University of Pernambuco (UPE). Research Coordinator of the Cardiac Emergency Hospital of Pernambuco (PROCAPE \\ - UPE), Fellow of the American College of Cardiology and the European Society of Cardiology, Recife, Brazil
}

The concept of palliative care (PC), first used at St. Joseph's Hospice for the Dying in 1905, has undergone a series of changes and extensions that allow it to be applied in the most varied clinical contexts. Although, until recently, PC was used only for patients with end-stage cancer, it is now applied to any serious (even if potentially reversible) disease that compromises the patient's and family's quality of life [1]. In this context it is now recognized that there are several possible needs for PC in heart failure (PAL-HF). Initially the time frame for the implementation of PC was in the terminal phase of the disease [2], characterized by functional class IV according to the New York Heart Association criteria. However, more recently the objectives of PAL-HF have been broadened, and several authors argue that, ideally, such care should accompany the patient from diagnosis to the end of life [3]. Therefore, the goal of PAL$\mathrm{HF}$ is to improve the quality of life of the patient/family, whether in the chronic course of HF or in HF with a faster progression.

However, what is observed in daily practice is a resistance (both by health professionals and by patients/family) to apply these concepts and initiate early PAL-HF, even with evidence showing its benefits in relation to quality of life, anxiety, spiritual well-being and depression when compared with standard treatment plus PC versus standard treatment alone in patients with HF [4], in addition to improved rates of survival [5]. A reason sometimes given to justify not-offering PC to patients with HF is the fact the end of life is not always easy to predict. We believe that this reflects the traditional idea, previously described, of reserving PC for patients already terminally ill. However, in addition to its importance at the end of life, PAL-HF decreases patients' suffering and distress, as well as affording a heightened, awareness of their condition preparing the patient/family dyad for a possible favorable outcome and helping them to see death as a natural and intrinsic process of the human condition.

In addition to the 'when' to start PAL-HF, another question still undefined and much discussed is the 'to whom' such care should be offered. Although a few decades ago, the provision of PC was practically restricted to oncologists and geriatricians, currently all health professionals need to be familiar with and know how to offer this care to the patient/family. The studies and applications of PC progressed so fast that it was necessary to create the specialty of palliative medicine, in which palliativists assume a prominent role in the multidisciplinary

Copyright: C2018 Goes GHB. This is an open-access article distributed under the terms of the Creative Commons Attribution License, which permits unrestricted use, distribution, and reproduction in any medium, provided the original author and source are credited. team. However, in developing countries such as Brazil, the vast majority of the population has very limited access to these professionals. Thus, it is up to the cardiologist, family physician, nurses and all other health professionals to include PC in the treatment of the patient, who needs to be prepared for it.

Another factor that hinders this access to the palliativist is the need to precisely define the moment at which the patient needs to be evaluated by the specialist, since HF is unpredictable and there are various indications for the consultation. We believe that, among the criteria mentioned in the literature, anxiety, depression, multiple hospitalizations due to HF decompensation and difficulty in pharmacological management are clear indications of the need for referral to a unit providing palliative care.

\section{Conclusion}

Despite important advances in the applicability of palliative medicine in cardiovascular disease, there are many aspects still to be investigated by researchers and applied in clinical practice. Palliative medicine, based on the holistic view, has been responsible for tremendous advances in the management of cancer patients, and increasingly robust evidence has appeared showing these benefits in the cardiopathy patients also. Thus, what is expected for the future in cardiology is the ever-increasing incorporation of PC in the follow-up of patients with chronic disease.

\section{References}

1. Sepulveda C, Marlin A, Yoshida T, Ullrich A (2002) Palliative care: The world health organization's global perspective. J Pain Symptom Manage 24: 91-96. [Crossref]

2. Hupcey JE (2012) The state of palliative care and heart failure. Heart Lung 41: 529530 [Crossref]

3. Hupcey JE, Penrod J, Fenstermacher K (2009) A model of palliative care for heart failure. Am J Hosp Palliat Care 26: 399-404. [Crossref]

4. Rogers JG, Patel CB, Mentz RJ, Granger BB, Steinhauser KE, et al. (2017) Palliative care in heart failure: The PAL-HF randomized, controlled clinical trial. $J$ Am Coll Cardiol 70: 331-341. [Crossref]

5. Connor SR, Pyenson B, Fitch K, Spence C, Iwasaki K (2007) Comparing hospice and nonhospice patient survival among patients who die within a three-year window. $J$ Pain Symptom Manage 33: 238-246. [Crossref]

${ }^{\star}$ Correspondence to: Gustavo Goes, Rua Arnóbio Marques, Santo Amaro, Recife, PE - Brazil, E-mail: gustavogoesmt@gmail.com

Received: October 08, 2018; Accepted: October 17, 2018; Published: October 19,2018 